\title{
Margherita Costa, la poetessa virtuosa
}

\section{Introduzione}

Tra le scrittrici "dimenticate" del secolo XVII, Margherita Costa (1600 ca.-1657) si distingue per la sua copiosissima e articolata produzione e per i toni, scanzonati e lucidi, con cui rivendica l'indipendenza femminile. La professione di cantante e virtuosa le consente di ottenere una certa autonomia e libertà nel contesto misogino seicentesco; la sua produzione risulta anomala a livello quantitativo e tematico sia se confrontata con quella delle contemporanee sia in quanto esempio di partecipazione attiva al consesso letterario in un contesto socio-politico in cui la scrittura femminile era in declino. Infatti, nel suo vastissimo corpus si possono riconoscere diversi generi: lirico, prosaico, storico, religioso, epistolare, drammatico (comico, mitologico, pastorale), attraverso i quali la poetessa non solo dimostra la sua versatilità, ma anche la capacità di usare registri diversi che oscillano tra il comico e il tragico, la satira e l'encomio, l'aulico e l'erotico. Eppure, l'intera produzione della Costa, nonostante gli elementi di novità e i messaggi moderni proposti, non ha goduto di fortuna critica. Anzi, «dal silenzio ha tutto da guadagnare» scriveva Dante Bianchi (1925: 211), condannando di fatto alla polvere degli scaffali dimenticati una delle più prolifiche e vivaci scrittrici del XVII secolo. Né meno impietosi sono stati i successivi lettori della virtuosa romana, come Martino Capucci che riconosceva alla sua copiosa produzione un interesse esclusivamente sociologico, perché «i soli tratti di qualche efficacia [sono] quelli dove erompe una aperta lubricità che si direbbe professionale» (CAPUCCI 1984, 233). Gli ultimi decenni, tuttavia, nell'ambito di una rinnovata attenzione critica alla scrittura femminile del XVII secolo, hanno segnato finalmente un'inversione di tendenza e i primi lavori monografici e le prime edizioni critiche hanno evidenziato la necessità di una riscoperta della poliedrica produzione della poetessa romana.

Questo numero monografico di «altrelettere» raccoglie gli atti del Convegno internazionale Margherita Costa, la poetessa virtuosa (L’Aquila, 12 aprile

The e-journal «altrelettere» is hosted at the URL: http://www.altrelettere.uzh.ch, in accordance with the Open Access Policy of the University of Zurich. Please cite this article as follows: D. DE LISO, M. Di MARO, V. MEROLA, Margherita Costa, la poetessa virtuosa, in «altrelettere», (2021), pp. 1-7, DOI: 10.5903/al_uzh-49.

(C) This article is licensed under a Creative Commons Attribution 2.5. Switzerland (CC BY-NC-ND 2.5). Please read the license terms on the website: http://creativecommons.org/licenses/by-nc-nd/2.5/ch/deed.en 
2021), organizzato da Daniela De Liso e Valeria Merola e promosso dal Dipartimento di Scienze Umane (Dipartimento di Eccellenza) dell’Università degli Studi dell'Aquila e dal Dipartimento di Studi Umanistici dell’Università degli Studi Federico II di Napoli. Il convegno avvia una rete internazionale di studi intorno alla figura e all'opera di Margherita Costa, inaugurando un dibattito interdisciplinare, e intercontinentale, tra studi letterari, teatrali e musicali. Lo scopo delle organizzatrici e delle studiose intervenute è di far scoprire e riscoprire una figura bollata dagli studi precedenti, mostrando il valore intrinseco della sua produzione - nel dialogo tra forme, generi e linguaggi diversi - e valutandone assunti ed apporti nel milieu letterario e culturale del XVII secolo.

Gli atti del Convegno saranno pubblicati in due fasi: in questa prima uscita sono raccolti i contributi di Natalia Costa-Zalessow, Daniela De Liso, Maria Di Maro, Valeria Merola e Clara Stella, che offrono una lettura dei vari momenti della produzione della romana. Costa-Zalessow tratteggia un quadro complessivo della produzione lirica di Costa, presentandone i temi muliebri innovativi. Di Maro propone una lettura macro-testuale dei primi due canzonieri costiani, La Chitarra e Il Violino, sottolineando i suoi debiti verso la tradizione e illustrando i punti di contatto con la weltanschauung mariniana e marinista. Stella offre una lettura del Cecilia Martire, ponendo l'attenzione sulle modalità di autorappresentazione di sé e celebrazione dei Barberini. De Liso presenta una dettagliata analisi delle figure femminili e dei motivi autobiografici ne La Selva di Diana. Merola, infine, si concentra sull'attività teatrale e mostra il continuo lavoro di riscrittura di Costa attraverso la lettura della sua ultima opera, Gli amori della luna. Gli atti del convegno saranno poi integrati in una seconda uscita che accoglierà i contributi di Monica Garcia Aguilar, Sara E. Diaz, Jessica Goethals, Teresa Megale e Julie Robarts, per completare il ritratto della poetessa virtuosa che il Convegno, prima, e questi Atti, poi, intendono offrire alla comunità di lettori.

A conclusione di queste poche battute di presentazione, intendiamo innanzitutto ringraziare le studiose, che da tre continenti diversi, hanno accettato il nostro invito e hanno contribuito a costruire un dialogo intenso intorno alla figura di Margherita Costa; le e i partecipanti, che spinte/i dalla curiosità, hanno seguito i lavori e hanno conosciuto la poetessa e colto il suo 
D. De Liso, M. Di Maro, V. Merola, Margherita Costa, la poetessa virtuosa, in «altrelettere», (2021), pp. 1-7, DOI: 3 10.5903/al_uzh-49.

valore nella storia culturale; e, infine, un sentito ringraziamento ad Adriana Chemello e Tatiana Crivelli per averci invitato a presentare i frutti del Convegno sulla loro rivista e permesso di pubblicare in tempi brevi i nostri lavori.

Daniela De Liso, Maria Di Maro, Valeria Merola 
D. De Liso, M. Di MARo, V. Merola, Margherita Costa, la poetessa virtuosa, in «altrelettere», (2021), pp. 1-7, DOI: 4 10.5903/al_uzh-49.

\section{Bibliografia}

Opere di Margherita Costa

CosTA 1630:

Margherita CostA, Istoria del viaggio d'Alemagna, Venezia, s.i.t., 1630.

CosTA 1638a:

Margherita CostA, La Chitarra, Francoforte, Daniel Watsch, 1638.

CosTA 1638b:

Margherita CosTA, Il Violino, Francoforte, Daniel Watsch, 1638.

CosTA 1639a:

Margherita CosTA, Lo Stipo, Venezia, s.i.t., 1639.

COSTA 1639b:

Margherita CosTA, Lettere amorose, Venezia, s.i.t., 1639.

CosTA 1640a:

Margherita Costa, Flora feconda, Firenze, Massi\&Landi, 1640.

CosTA 1640b:

Margherita Costa, Flora feconda. Dramma, Firenze, Massi\&Landi, 1640.

COSTA 1640c:

Margherita Costa, La Selva di Cipressi, Firenze, Massi\&Landi, 1640.

CosTA 1641:

Margherita CostA, Li Buffoni, Firenze, Massi\&Landi, 1641.

CosTA 1644:

Margherita CosTA, Cecilia martire, Roma, Mascardi, 1644.

CosTA 1647a:

Margherita Costa, La Selva di Diana. Opera di Margherita Costa Romana, dedicata all'Altezza Reale di Madama di Savoia, A Parigi, per Sebastiano Cramoisy, stampatore ordinario del Re, e della Regina Regente, 1647

COSTA 1647b:

Margherita CostA, La tromba di Parnaso, Parigi, Craimoisy, 1647.

CosTA 1647a:

Margherita Costa, Festa reale per balletto a cavallo, Parigi, Craimoisy, 1647.

COSTA 1654:

Margherita CostA, Gli amori della luna, Venezia, Giuliani, 1654. 
D. De Liso, M. Di MARo, V. Merola, Margherita Costa, la poetessa virtuosa, in «altrelettere», (2021), pp. 1-7, DOI: 5 10.5903/al_uzh-49.

\section{Saggi critici:}

AGUILAR 2014:

Mónica García AGUILAR, Margherita Costa y la comedia bufonesca del siglo XVII, in Escritoras italianas fuera del canon, a c. di Daniele Cerrato, Sevilla, Benilde, 2017, pp. 181-96.

\section{AGUILAR 2019:}

Los bufones: comedia ridícula di Margherita Costa, a c. di Mónica García Aguilar, Sevilla (España), Arcibel Editores, 2019.

BELLESI 2018:

Sandro BELLESI, I ritratti delle sorelle Costa di Cesare Dandini e Stefano della Bella, in Con dolce forza: donne nell'universo musicale del Cinque e Seicento, Firenze, Polistampa, 2018, pp. 65-73.

\section{BIANCHI 1924-1925:}

Dante BIANCHI, Una cortigiana rimatrice del Seicento: Margherita Costa, in «Rassegna critica della letteratura italiana», 29 (1924), 1-31, pp. 187-203; 30 (1925), pp. 158-211.

BROSIUS 2008:

Amy BRosius, 'Il suon, lo sguardo, il canto': The Function of Portraits of MidSeventeenth-Century "Virtuose" in Rome, in «Italian studies», 1 (2008), pp. 17-39.

CAPUCCI 1984:

Martino CAPPUCCI, Costa, Margherita, in Dizionario biografico degli italiani, Roma, Enciclopedia Treccani, 1984, vol. 30, pp. 232-34.

COLLER 2017:

Alexandra COLLER, Margherita Costa's Li buffoni (1641): The First (Extant) Female-Authored Scripted Comedy Women, in Women, Rhetoric, and Drama in Early Modern Italy, London \& New York, Routledge, 2017, pp. 41-72.

\section{DIAZ-GOETHALS 2018:}

Margherita COSTA, The buffoons: a ridiculous comedy. A bilingual edition, a c. di Sara E. Díaz, Jessica Goethals, Toronto, Arizona Center for Medieval and Renaissance Studies, 2018.

COSTA-ZALESSOW 1982:

Natalia Costa-ZALESSOW, Scrittrici italiane dal XIII al XX secolo: testi e critica, Ravenna, Longo, 1982.

COSTA-ZALESSOW 2008:

Natalia CosTA-ZALESSOW, Margherita Costa, in Albert N. MANCINI, Glenn Palen PIERCE, Dictionary of Literary Biography. Seventeenth-century Italian poets and dramatists, Bruccoli, Clark Layman, 2008, vol. 339, pp. 113-18.

\section{COSTA-ZALESSOW 2010:}

Natalia COSTA-ZALESSOW, Una poesia femminista del 1672 anonima e dimenticata, da attribuire a Margherita Costa, in «Esperienze letterarie», 35 (2010), 4, pp. 7985 . 
D. De Liso, M. Di MARo, V. Merola, Margherita Costa, la poetessa virtuosa, in «altrelettere», (2021), pp. 1-7, DOI: 6 10.5903/al_uzh-49.

COSTA-ZALESSOW 2015:

Natalia COSTA-ZALESSOW, Voice of a Virtuosa and Courtesan. Selected poems of Margherita Costa, New York, Bordighera Press, 2015.

COSTA-ZALESSOW 2019:

Natalia Costa-ZALESSOW, Cortegiano ravveduto: satira di Margherita Costa, in «Letteratura italiana antica: rivista annuale di testi e studi», XX (2019), pp. 579-91.

Cox 2008:

Virginia Cox, Women's writing in Italy 1400-1650, Baltimore, John Hopkins UP, 2008.

Cox 2011a:

Virginia CoX, Declino e caduta della scrittura femminile nell'Italia del Seicento, in Verso una storia di genere della letteratura italiana, a c. di Virginia Cox e Chiara Ferrari, Bologna, Il Mulino, 2011, pp. 157-84.

Cox 2011b:

Virginia Cox, The prodigious Muse. Women's writing in Counter-Reformation Italy, Baltimore, John Hopkins UP, 2011.

CROCE 2003:

Benedetto CROCE, Donne letterate nel Seicento, in Nuovi saggi sulla letteratura italiana del Seicento, Napoli, Bibliopolis, 2003, pp. 165-82.

DE LISO 2020:

Daniela DE LISO, Le lettere amorose di Margherita Costa, in (Auto)narrativas: hacia la construcción de un canon alternativo en italiano, a c. di Sara Velázquez García e Laureano Núñez García, Salamanca, Ediciones Universidad de Salamanca, 2020, pp. 47-64.

DI MARO 2019:

Maria Di MARo, Il Violino di Margherita Costa: prime indagini, in Escritoras italianas inéditas en la querella de las mujeres: traducciones en otros idiomas, perspectivas y balances, a c. di Salvatore Bartolotta e Mercedes Tomo-Ortiz, Madrid, UNED editorial, 2019, vol. II, pp. 43-56.

DI MARO 2020:

Maria DI MARO, Una poetessa del XVII secolo: Margherita Costa, in (Auto)narrativas: hacia la construcción de un canon alternativo en italiano, a c. di Sara Velázquez García e Laureano Núñez García Salamanca, Ediciones Universidad de Salamanca, 2020, pp. 81-97.

FERRONE 1986:

Siro FERRONE, Nota biobibliografica, in Commedie dell'arte, Milano, Mursia, 1986, 2 voll., II, pp. 235-38.

GOETHALS 2017a:

Jessica GOETHALS, The Bizarre Muse: the literary Persona of Margherita Costa, in «Early modern women», 12 (2017), 1, pp. 48-72.

GOETHALS 2017b:

Jessica GoEthals, The Patronage Politics of Equestrian Ballet, in «Renaissance Quarterly», 70 (2017), 4, pp. 1397-48. 
D. De Liso, M. Di MARo, V. Merola, Margherita Costa, la poetessa virtuosa, in «altrelettere», (2021), pp. 1-7, DOI: 7 10.5903/al_uzh-49.

GOETHALS 2020a:

Jessica GOETHALS, The singing saint: the martyrdom of saint Cecilia in Seventeenth-century literature and theatre, in «Women language literature in Italy: Donne lingua letteratura in Italia», 2 (2020), pp. 43-61.

GOETHALS 2020b:

Jessica GoETHALS, Worth Its Salt: Margherita Costa's Ridiculous Defence of Buffoonery, in «The Italianist», 40 (2020), 3, pp. 362-81.

MARONGIU 2021:

Paola MARONGIU, Margherita Costa: una scrittrice femminista del XVII secolo, in «Critica letteraria», 190 (2021), 1, pp. 115-33.

MEGALE 1988:

Teresa MEgale, La commedia decifrata. Metamorfosi e rispecchiamenti in $\mathrm{Li}$ Buffoni di Margherita Costa, in «Il castello di Elsinore», 2 (1988), pp. 64-76.

PIANTONI 2017:

Luca PIANTONI, L'epistolario amoroso di Margherita Costa, in L'Italianistica oggi: ricerca e didattica, Atti del XIX Congresso dell'ADI - Associazione degli Italianisti (Roma, 9-12 settembre 2015), a c. di B. Alfonzetti, T. Cancro, V. Di Iasio, E. Pietrobon, Roma, Adi editore, 2017, pp. 1-5.

PIANTONI 2018:

Luca PiANTONI, Le lettere amorose di Margherita Costa tra sperimentalismo e 'divertissement', in «Studi Secenteschi», 59 (2018), pp. 33-52.

ROBARTS 2019:

Julie L. ROBARTS, Challenging Male Authored Poetry: Margherita Costa's Marinist Lyrics (1638-1639), Tesi di dottorato, The University of Melbourne, Australia, 2019.

SALVI 1997:

Marcella SALVI, "Il solito è sempre quello, l'insolito è più nuovo": "Li buffoni" e le prostitute di Margherita Costa fra tradizione e innovazione, in «Forum Italicum», 2 (2004), pp. 376-99. 